\title{
Pelvic heterotopic ossification: when CT comes to the aid of MR imaging
}

\author{
Andrea Zagarella • Elisabetta Impellizzeri • Roberta Maiolino • \\ Raffaele Attolini • Maria Chiara Castoldi
}

Received: 18 April 2013 / Accepted: 4 June 2013 / Published online: 5 July 2013

(C) The Author(s) 2013. This article is published with open access at Springerlink.com

\begin{abstract}
Objective This article compares various imaging aspects of magnetic resonance (MR) and computed tomography (CT) of heterotopic ossification (HO) in the pelvic soft tissues in paraplegic patients. Our aim is to highlight the benefits of integrating MR and CT imaging in the diagnosis of immature HO, which may be challenging with MR images alone.

Methods Paraplegic patients examined on the same day by contrast-enhanced 0.4-T pelvic MR and unenhanced CT for pressure-sore-related infections were selected. MR imaging was performed on a Hitachi-Aperto 0.4 T; the Open Magnet served as a more favourable configuration for the required limb positioning of these patients. CT images were attained on a six-slice Siemens-Somaton-Emotion.

Results MR images of $\mathrm{HO}$ differ according to the degree of bone maturity. The more immature the HO process, the more heterogeneous is the signal, characterised mostly by focal iso-hypointensity on T1-weighted images and hyperintensity on T2-weighted/short TI inversion recovery (STIR). These characteristics correlate to different CT patterns.

Conclusions MR and CT features of pelvic HO in paralysed patients were reviewed with a focus on the different aspects associated with the degree of ossification. Based solely on the MR findings, immature heterotopic ossification may be difficult to differentiate from other soft tissue pelvic lesions. Teaching points

- The pelvis and hip are common locations of heterotopic ossifications (HO), often occurring in paraplegic patients.
\end{abstract}

A. Zagarella $(\bowtie) \cdot$ E. Impellizzeri $\cdot$ R. Maiolino $\cdot$ M. C. Castoldi Radiology Department, Istituti Clinici di Perfezionamento di Milano, C.T.O., Via Bignami 1, 20162 Milan, Italy

e-mail: andrea.zagarella@icp.mi.it

R. Attolini

Paraplegic Unit - Orthopedic Surgery Department, Istituti Clinici di Perfezionamento di Milano, C.T.O., Via Bignami 1, 20162 Milan, Italy
- With respect to $H O, M R$ imaging allows for a confident diagnosis in mature ossified lesions only. The MR aspect of immature ossification may be confused with other pathologies.

- Plain radiographs and CT may show various phases of ossification: amorphous calcification, immature and mature ossification.

- Integrating MR with CT can help recognise HO foci and differentiate them from infections and other soft tissue lesions.

Keywords Musculoskeletal $\cdot$ Neuro $\cdot \mathrm{CT} \cdot \mathrm{MR}$

\section{Introduction}

Heterotopic ossification is the development of bone in abnormal sites. It may originate at any extraskeletal location where undifferentiated mesenchymal cells are found.

While its etiopathogenesis is unknown, it is likely that more than one factor (vascular stasis, low tissue oxygenation, microtraumas) contributes to activation of osteoblast and chondroblast progenitor cells with a consequent precipitation of calcium salts within the connective tissue [1-3]. Clinically, heterotopic ossification (HO) occurs in rare congenital disorders and, more commonly, in acquired conditions such as after surgery or related to spinal cord or head lesions. This latter condition, called neurogenic heterotopic ossification, is the topic of this article.

Ossifying lesions run along an ossification continuum: amorphous calcification, to immature ossification, to mature ossification.

MR imaging alone allows easy recognition of mature ossifications; however, amorphous calcification or immature ossification shows non-specific signal and contrast enhancement features. Thus, since enhanced MR imaging is routinely performed on paraplegic patients with infection of the 
Fig. 1 A 58-year-old paraplegic man, post trauma. a On axial STIR a diffuse increased signal intensity involving the right obturator externus-adductor muscles suggests an inflammatory reaction as observed in the early phase of ossification. b On CT axial scan a circumscribed amorphous calcification showing ill-defined margins and no trabecular structure is evident within the periphery of the adductor brevis muscle belly
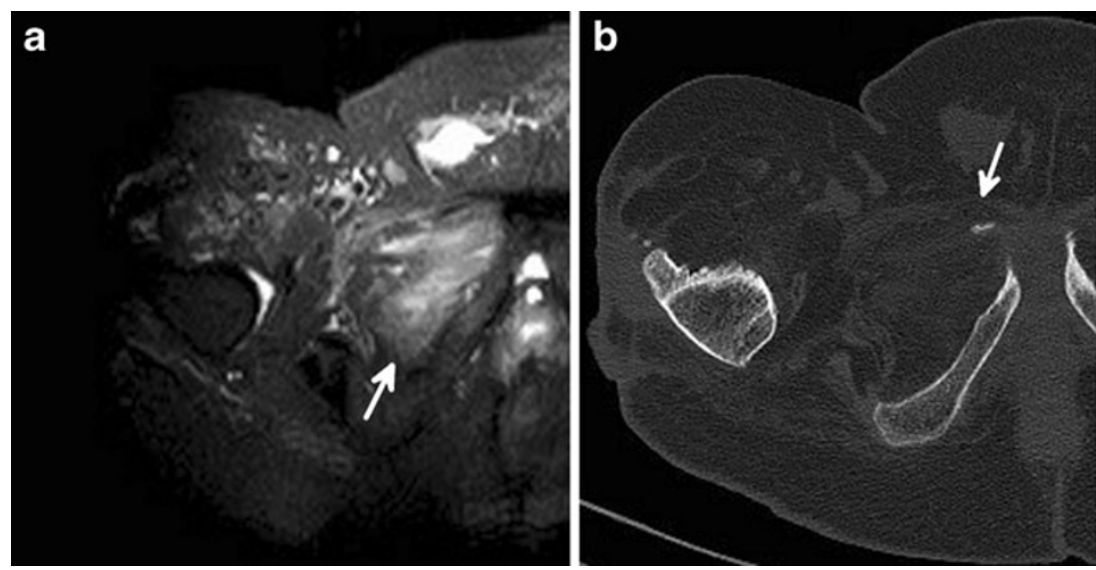

pelvis, the differential diagnosis between $\mathrm{HO}$ and other soft tissue diseases can be problematic. Plain radiographs and CT can help in identifying the ossifying process showing peculiar morphological characteristics related to the degree of maturity.

In this review article, we describe our experience with pelvic imaging in paraplegic patients. We focus on different $\mathrm{HO}$ features through a simultaneous comparison of MR and CT findings.

All examined patients underwent both a contrast-enhanced MR scan and an unenhanced CT scan on the same day. All patients were scanned on a 0.4-T open magnet to accommodate required patient positioning. Our goal in this review is to underscore the importance of integrating $\mathrm{MR}$ imaging with $\mathrm{CT}$ to differentiate $\mathrm{HO}$ from other processes in the clinical management of paraplegic patients. More generally, integrated imaging can be helpful whenever an ossifying process may be expected in the pelvis and/or around hip joints.

\section{HO in paraplegia}

$\mathrm{HO}$ is frequently observed in paraplegic patients with spinal cord lesions of traumatic or atraumatic origin. Its incidence varies from $15 \%$ to $35 \%$ [1-3]. This phenomenon is mostly observed about larger joints, hips being the more affected site.

The cause of HO in paraplegia is unknown. Tissue denervation, oedema, and vascular stasis are all considered related to the formation of $\mathrm{HO}$ that usually occurs without precipitating trauma. Nevertheless, some authors have hypothesised that microtrauma may play a role in the etiopathogenesis of neurogenic $\mathrm{HO}$ stemming from passive physiotherapy joint manipulation [4].

Initial clinical findings of $\mathrm{HO}$ relate to the inflammatory process, and are characterised by local swelling, redness, muscle spasms and pain. Early in the clinical setting HO may be confused with infection, fracture or neoplasia [3]. The inflammatory process then evolves to the formation of a
Fig. 2 A 55-year-old tetraplegic male patient following vertebral trauma. a Axial CT scan shows a bilateral ossifying process about the femural metaphyseal region with some amorphous calcifications in the ileopsoas muscle area. A left throcanteric calcific bursitis is also observed. b-d MR imaging: (b) axial STIR and (c) T1-weighted axial images show bilateral ileopsoas bursitis (small arrows) on the right merging to a mature ossification. d T1weighted fat-suppressed images after contrast administration show, on the right side, peripheral enhancement of the bursal walls (large arrow)
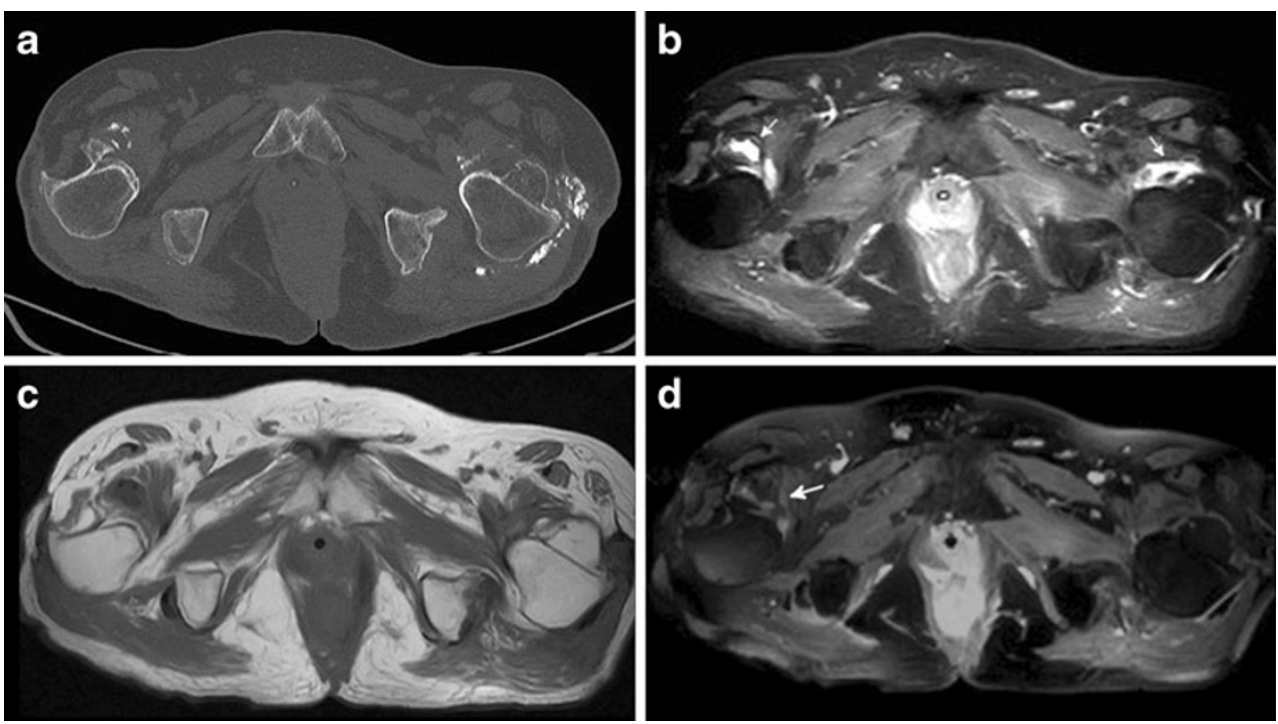


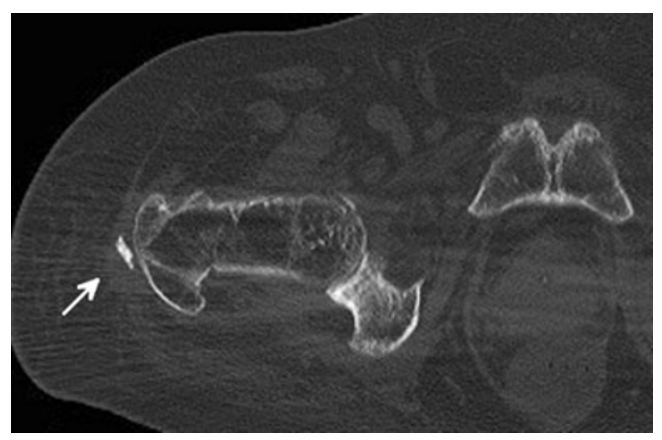

Fig. 3 A 62-year-old male patient with traumatic paraplegia. CT imaging shows soft-tissue thickening adjacent to the great trochanter due to bursitis. An immature ossification with an initial trabecular structure and faint margins is evident within the paratrochanteric bursa

connective mass with progressive deposition of bone tissue. In this phase, HO can cause motion restriction, joint ankylosis and decubiti. In fact, as many as $5-10 \%$ of patients develop complete hip ankylosis $[1,2,4,5]$. Typically, HO develops between 1 and 5 months after spinal injury, and can continue to be active even 5 years after the onset of paraplegia [6]; in some cases, it never reaches complete maturity [7]. On radiographic imaging, HO foci vary in size and shape, and can range from a thin, 1-mm-long strip to a mass of many centimetres in diameter.

\section{HO distribution and sites of origin}

The most common sites of neurogenic pelvis ossification are the iliac, ileopsoas or vastus muscle areas [7].

According to the literature, the initial $\mathrm{HO}$ process can originate inside a muscle or extend into a bursa [2]. In cases of primary muscle involvement, initial ossification starts in the peripheral tissue due to oedema and capillary stasis following muscle denervation [3]. The pathogenesis of $\mathrm{HO}$ is similar to that of circumscribed myositis ossificans [8-10]; therefore, it manifests many of the same imaging features, including T2/short TI inversion recovery (STIR) hyperintensity and some peripheral enhancement. Muscles initially involved in the ossification process show diffuse hyperintensity on T2/STIR images with or without some heterogeneity within them. The corresponding CT appearance consists of a certain degree of swelling of the muscle, which can contain some peripheral calcification or immature ossification (Fig. 1).

Bursae are other sites where HO can take place initially and progress to compression of the adjacent muscles [11]. Some areas of elevated signal intensity on T2-weighted images with rim contrast enhancement may represent fluidfilled merged bursae or inclusion within the ossification itself (Fig. 2). In cases of primary bursal involvement, it is not always possible to differentiate $\mathrm{HO}$ from pressure-related calcified bursitis that can evolve in ossification [2] (Fig. 3).

Our patient population showed immature $\mathrm{HO}$ either in the muscle and fascia or in the bursae; however, we never found mature HO limited to the bursae. We assume that with increased maturity, $\mathrm{HO}$ formations tend to coalesce and adhere to the contiguous bone, which fades anatomic landmarks. CT imaging can easily identify calcium deposits inside a muscle or calcification/ossification at the anatomical site of a bursa, as is usually observed in initial soft tissue ossification.

\section{Imaging findings}

Imaging can assist in the identification of three degrees of HO: amorphous calcifications, immature calcifications and mature calcifications [7].

Both plain radiographs and $\mathrm{CT}$ imaging are the standard references to assess $\mathrm{HO}$ maturity, since CT more accurately defines the different stages of ossification than do plain radiographs.

Amorphous calcification is poorly defined with no recognisable trabecular structure (Fig. 4a); immature ossification
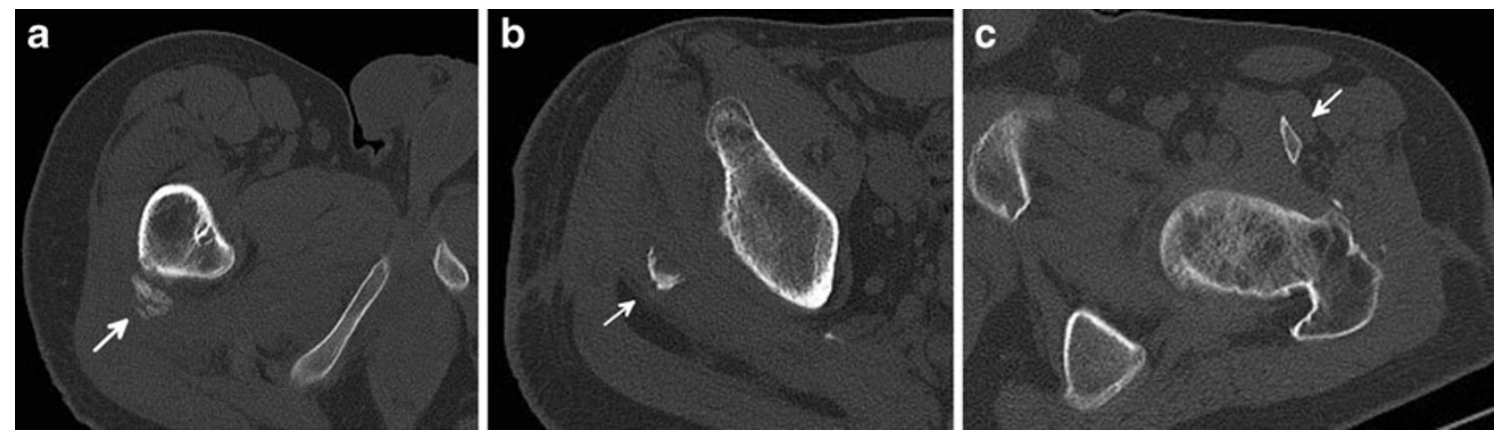

Fig. 4 Different patterns of HO maturity shown on CT. a Amorphous calcification located within the right quadratus femoris muscle, characterised by ill-defined margins and no recognisable trabecular structure. b Immature ossification located within the right medius gluteus muscle. Lesion shows poorly-defined margins and an initial trabecular formation is recognisable. c Mature ossification located within the left ileopsoas muscle, outlined by cortex with well-defined cancellous bone inside 
Fig. 5 A 72-year-old

tetraplegic male patient with spinal injury. Different grades of maturity may coexist in the same clinical setting. a Axial CT scan shows an $\mathrm{HO}$ formation located within the gluteus minimus muscle with the typical features of maturity: wellstructured cancellous bone outlined by cortex and adherent to cortical bone. Immature ossification and amorphous calcifications coexist in various regional muscles. b-d Axial corresponding MR imaging shows the mature ossification with a high-fat bone marrow signal outlined by low signal cortical bone (c) (small arrow). The amorphous calcifications contiguous to the posterior iliac bone (large arrow) are characterised by elevated signal intensity on STIR images

(b) and intense contrastenhancement on T1-weighted fat-saturated (d) compared with unenhanced T1-weighted images (c). The small immature ossification in the gluteus medius muscle (asterisks) is clearly demonstrated by CT (a), while it is not easily recognisable on MR imaging
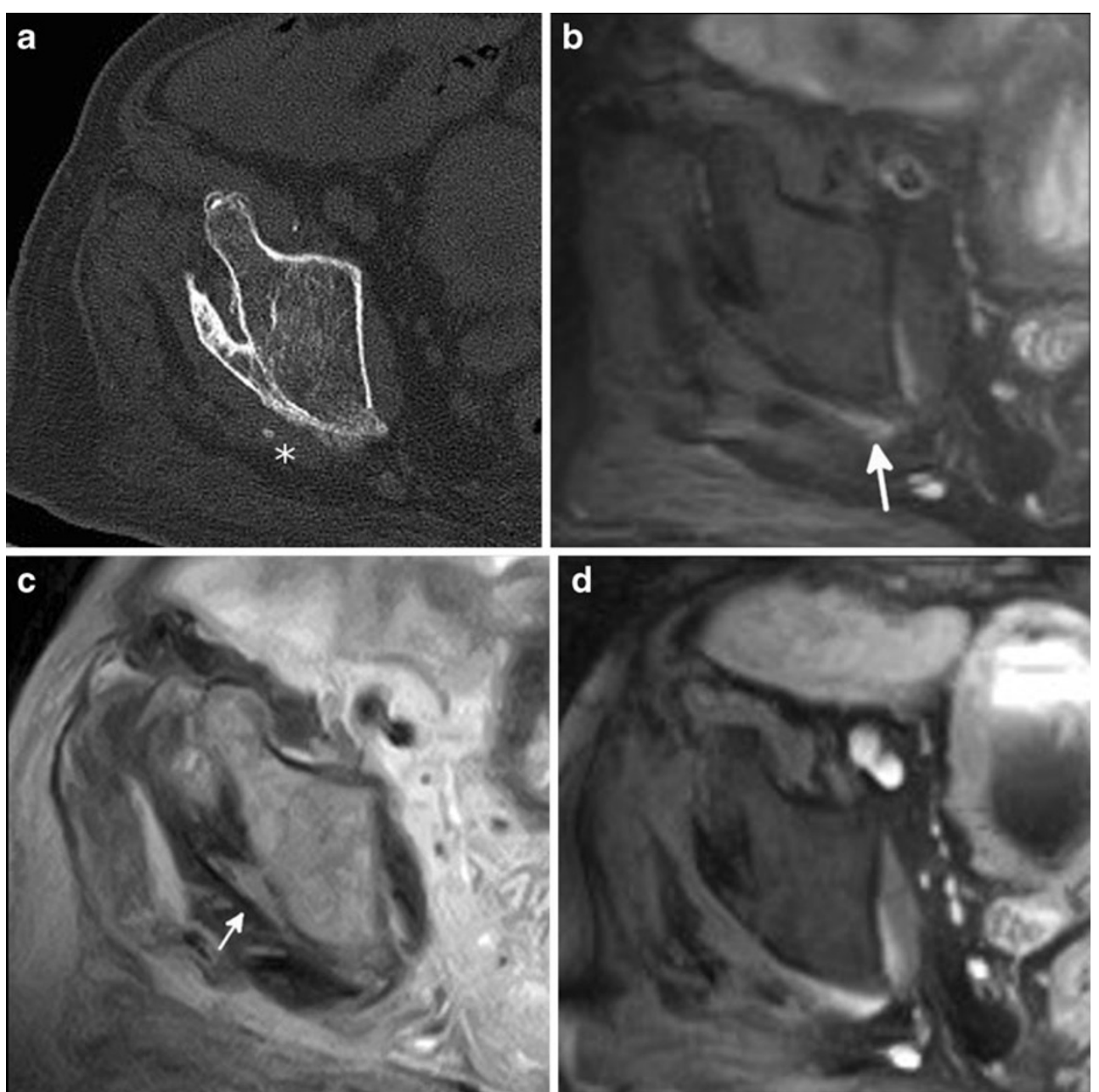

shows poorly defined margins and initial trabecular formation (Fig. 4b); mature ossification is characterised by a cortical outline with a well-defined cancellous bone centre (Fig. 4c). The morphological difference between amorphous calcification and immature ossification is not always sharp, and different degrees of maturity often coexist (Fig. 5).

At MR imaging, mature $\mathrm{HO}$ presents as typical cancellous fat bone that is hyperintense on T1- and T2-weighted images, outlined by a rim of hypointense cortical bone. At CT, this finding corresponds to bone formation characterised by cortical and trabecular structure. Amorphous calcification and immature $\mathrm{HO}$ can present non-specific signal intensity and contrast enhancement characteristics [2]. In cases of bone process immaturity, the formation can show hetereogeneous signal, characterised mostly by focal iso-hypointensity on T1weighted images and hyperintensity on T2-weighted/ STIR images, with occasional fluid contents. A certain amount of enhancement after contrast administration can occur both inside and peripheral to the lesion (Figs. 6, 7).

While these findings may present in various combinations (Figs. 8, 9), several conclusions can be formulated. The more immature the HO process, the more these non-specific signs
Fig. 6 A 35-year-old male paraplegic patient after spinal injury. a First CT axial scan shows swelling and hypodensity of the right ileopsoas muscle. b One-year follow-up examination shows the appearance of an immature ossification within the ileopsoas muscle
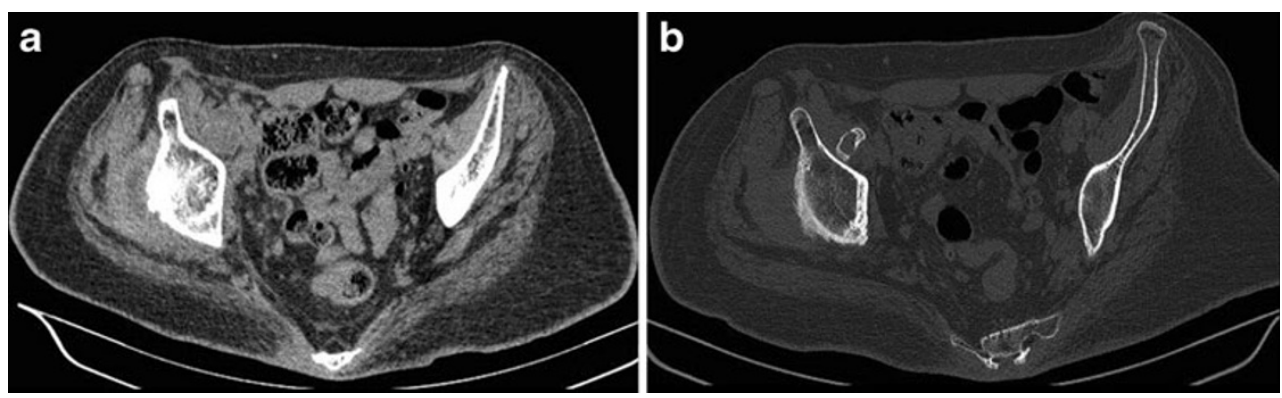
Fig. 7 The same patient as in Fig. 6: the corresponding MR examination $(\mathbf{a}, \mathbf{b})$ performed on the same day as CT examination shown in Fig. 6b. Muscle swelling and heterogeneity (large arrow) is clearly noticeable on STIR images (a) and T2-weighted images (b) associated with inflammatory changes of contiguous retroperitoneum tissue (asterisks). A marked intra-articular fluid collection is also evident to the right of the coxofemural joint (small arrows). T1-enhanced (d) compared with $\mathrm{T} 1$-weighted unenhanced images (c): after i.v. gadolinium administration, a heterogeneous and prominent contrast-enhancement is evident inside the right ileopsoas muscle encircling a "dark spot" corresponding to the ossification


Fig. 8 A 41-year-old male paraplegic following spinal trauma. a CT axial scan defines a bulky calcific mass located within the anterior compartment of the right thigh and the gluteus minimus muscle. On MR images (b-d), the mass is characterised by heterogeneity on STIR (b) and T1-weighted (c) images. d Focal contrastenhancement appears on T1weighted imaging following i.v. gadolinium administration (arrows)
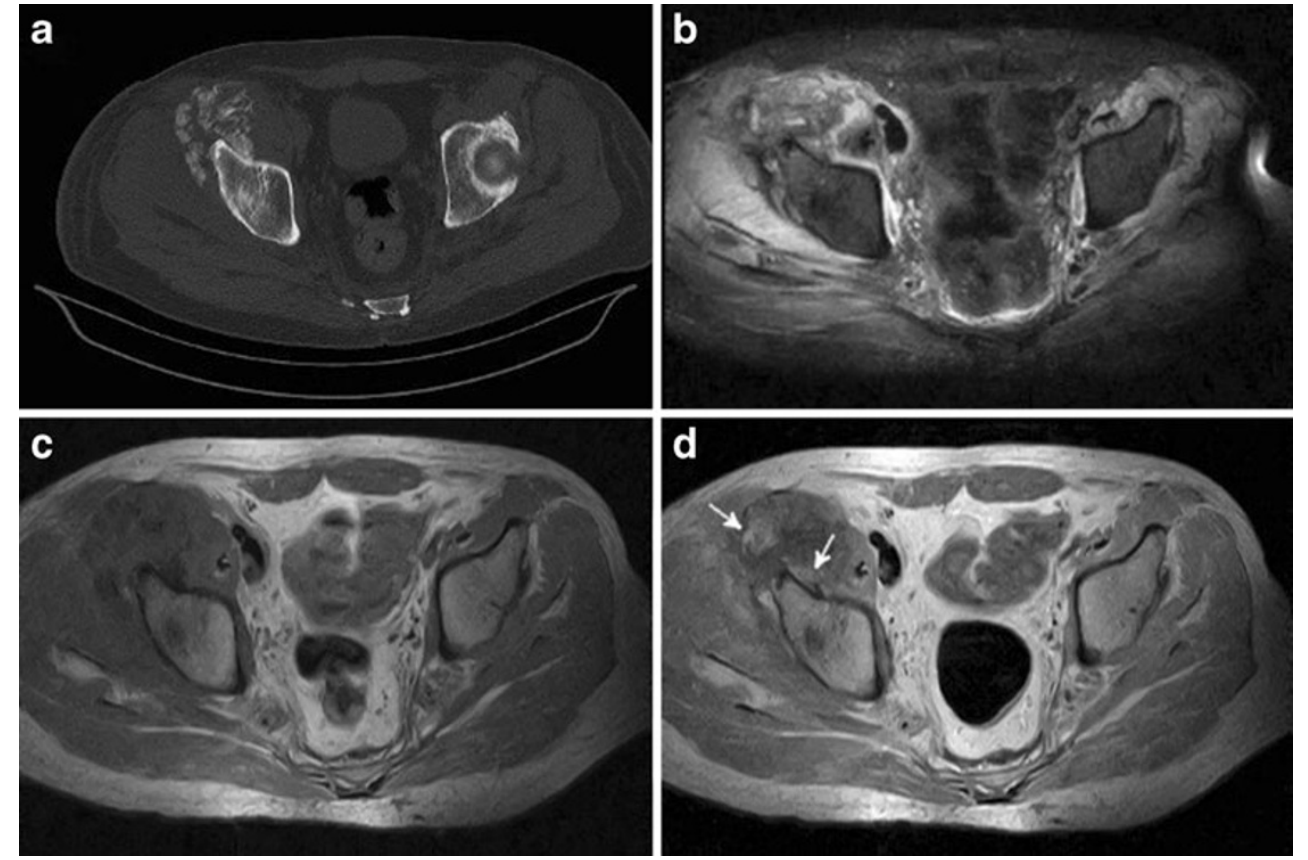
are observed [2]. Similar MR intensity characteristics can also be found in tumours, local infections and, if fluid is present, in abscesses.

When MR imaging reveals a lesion with an indeterminate aspect, CT can aid the diagnosis of HO by detecting amorphous calcification or immature ossification inside the lesion.

\section{Differential diagnosis and pitfalls}

Pelvic imaging of paraplegic patients may reveal masses of different natures, infection being the main pathology to be differentiated from HO. Soft tissue infections and abscesses often develop in paraplegic patients beneath deep pressure sores [12]. Their evaluation is performed primarily with MR imaging. As MR imaging can show lesions of different natures, the possibility of misleading features in immature $\mathrm{HO}$ should be considered during evaluation of a pelvic MR examination performed for an infection process. In these cases, CT imaging is helpful as it reliably detects the initial formation of trabecules within calcific lesions [13]. Nevertheless, the diagnosis of the nature of a lesion containing amorphous calcifications is not always obvious because dystrophic calcification may sometimes be present in soft tissue infectious processes or in septic bursitis [14] (Fig. 10).

Whenever a certain degree of uncertainty is present in defining the nature of the calcifying lesion, and the clinical setting allows a "wait and see" behaviour, carefully timed CT imaging can be helpful. In cases of sterile HO, follow-up CT can show the initial formation of a trabecular structure, which is a typical aspect of $\mathrm{HO}$ in evolution to maturity (Fig. 11). In cases of infection, CT imaging may show some changes in the ossified mass, such as a more pronounced fragmentation or even a burst ossification, which defines the diagnosis (Fig. 12).

Furthermore, as shown in Figs. 9 and 10, soft tissue infection and ossification formation may show peculiar behavioural patterns with respect to the superficial tissue. In paralysed patients, deep infection of the soft tissues is usually contiguous to skin ulcers and is always associated with inflammation of subcutaneous tissue since infection spreads through the skin [12]. Conversely, HO originates from deep
Fig. 9 A 61-year-old male paraplegic patient following spinal injury. a CT axial scan reveals an elongated ossifying lesion within the right pectineus muscle. b On STIR images the lesion (small arrow) is essentially isointense to muscle, while on (d) T1-weighted images it shows a considerable low signal intensity). c No contrast-enhancement is noticeable at this site. On the left ischiatic region, a deep infected soft tissue ulcer with bone involvement is shown (large arrow)
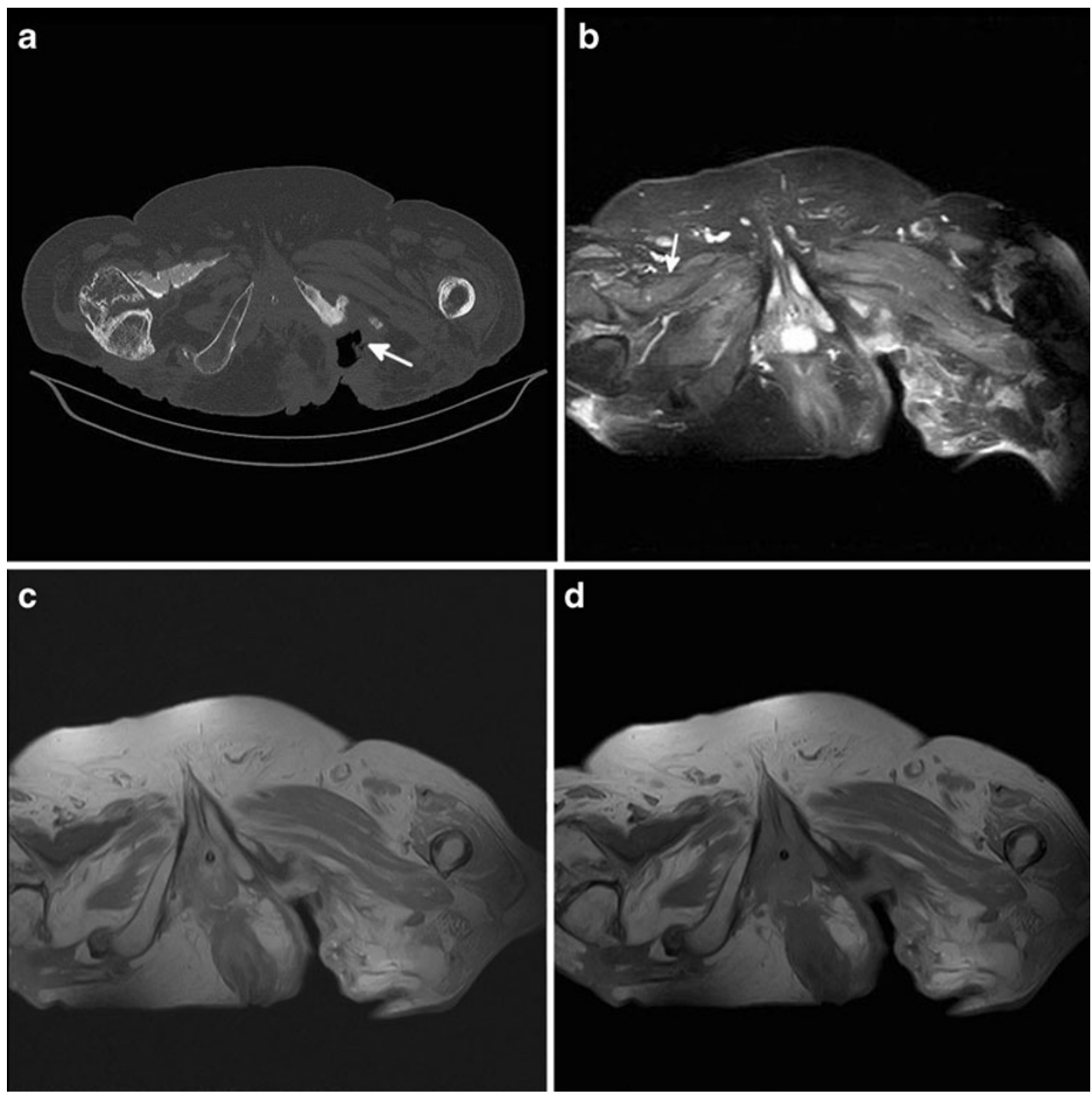
Fig. 10 A 35-year-old male patient with leg contractures, affected by posterior element incomplete fusion (spina bifida occulta). a MR T1-weighted axial images evidence a soft tissue abscess (small arrow) of the perineal region related to pressure sores. Lesion is determining a certain retraction over the superficial layers of the dermis. b STIR images show the focal inflammatory lesion partially filled with fluid and a large bursitis of the right gluteal region (large arrow). c On coronal T1-weighted fat-sat images after gadolinium administration, the abscess walls appear highly vascularised. The gluteal bursitis seems to be communicating with the infectious process, suggesting a septic bursitis. A prominent contrast enhancement is also seen to the sacrum, suggesting a bony involvement by the septic process. d Axial CT imaging performed with patient in a different leg positioning. The exam validates the connection between the perineal abscess and the gluteal bursitis. Amorphous calcifications (asterisks) are enclosed within the septic bursitis at the right gluteal region


in soft tissues; involvement of superficial subcutaneous layers is secondary to fibrotic retractive changes.

Contrary to immature $\mathrm{HO}$, mature $\mathrm{HO}$ is easily recognisable by MR imaging since its signal intensity is similar to that of normal cancellous bone outlined by cortex. When these features of $\mathrm{HO}$ appear on MR images, CT is not required, as it offers no additional information.

\section{Treatment}

HO may lead to a series of complications, including nerve entrapment, pressure ulcers and joint ankylosis. Consequently, early disease detection is extremely important.

Diphosphonates and non-steroidal anti-inflammatory drugs (NSAIDs) are used for prohylaxis or HO treatment [15].

After diagnosis, passive range-of-motion exercises to maintain joint mobility are prescribed [16].

When the ossification formation restricts mobility, surgical resection may be performed to increase joint function. During surgery planning, assessment of the degree of ossification is crucial, as resection must be performed only when $\mathrm{HO}$ is mature. Resection of an ossification process that has reached maturity implies less intra-operative complications, such as haemorrhage or a minor risk of post-operative recurrence [7]. On the other hand, excessive surgical delay may expose the patient to development of joint ankylosis. Imaging plays an important role in optimising the timing of surgery, and plain radiographs and $\mathrm{CT}$ imaging are the most reliable ways to evaluate advanced ossification foci.

\section{Conclusion}

Pelvic MRI of paraplegic patients is a diagnostic challenge. Based on MR findings, immature $\mathrm{HO}$ may be difficult to differentiate from other pathologies, such as soft tissue pelvic infection, abscess and septic bursitis. The radiologist should be aware of the different aspects of $\mathrm{HO}$ at MR imaging. Correlation between MR and CT features is critical to accurate diagnosis. When dubious findings are present, 
Fig. 11 A 34-year-old paraplegic male following spinal cord injury. Examinations were performed to evaluate abscess and osteomyelitis of the left ischial tuberosity (large arrow). The MR study reveals a lesion in the area of the right psoas muscle (small arrow): (a) central hypo-intense area on axial T1-weighted non-

enhanced image (small arrow), (b) enhanced tissue and some fluid after contrast administration (small arrow) Note that the sterile HO is similar to the abscess of the left ischial region. c The corresponding $\mathrm{CT}$ shows the mass as non-homogeneous with some subtle calcifications on the medial side. $\mathbf{d}$ The CT study repeated 10 months later shows transformation of the immature bone into a mature, heterotopic ossification that adheres to the femur
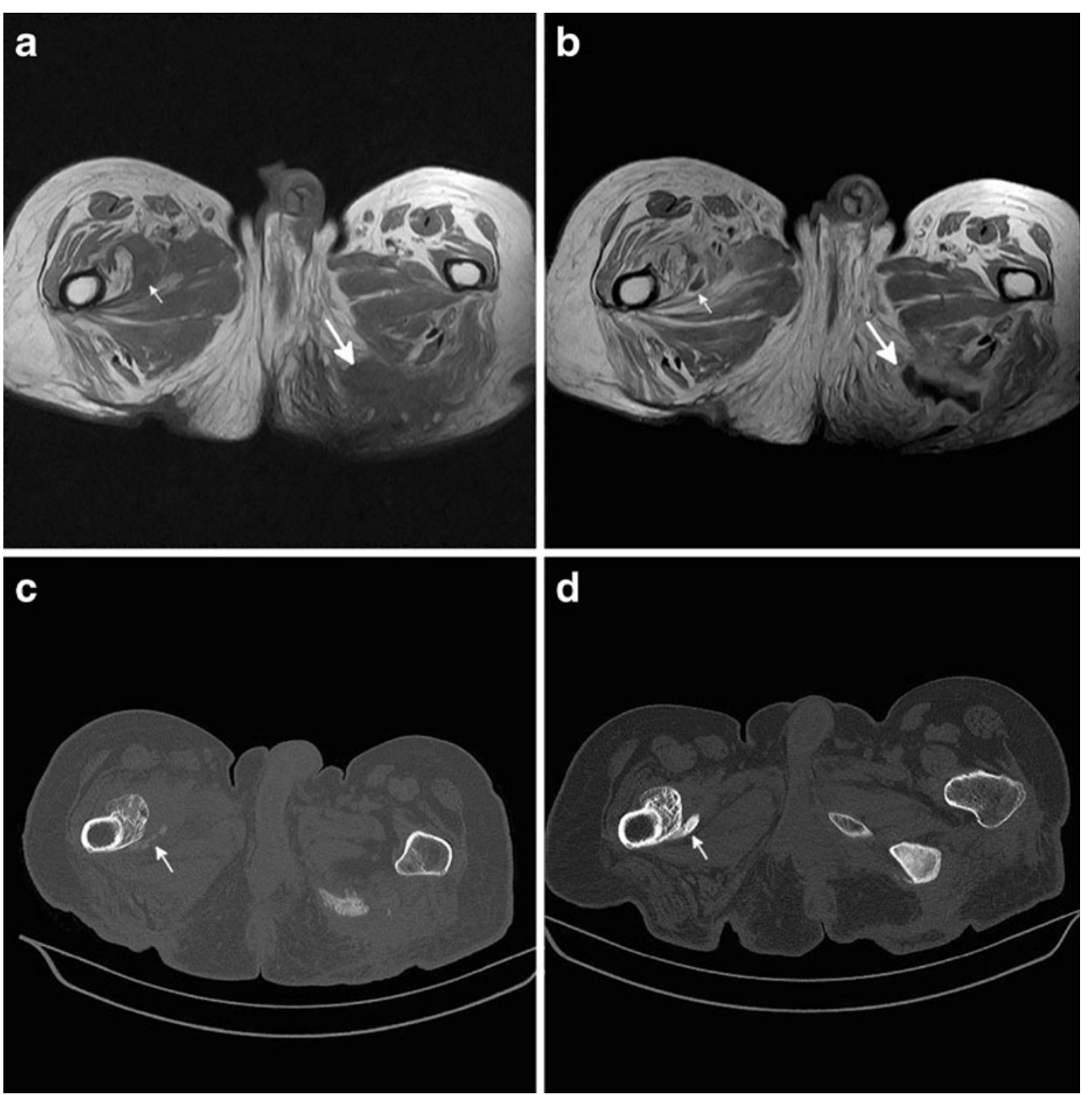

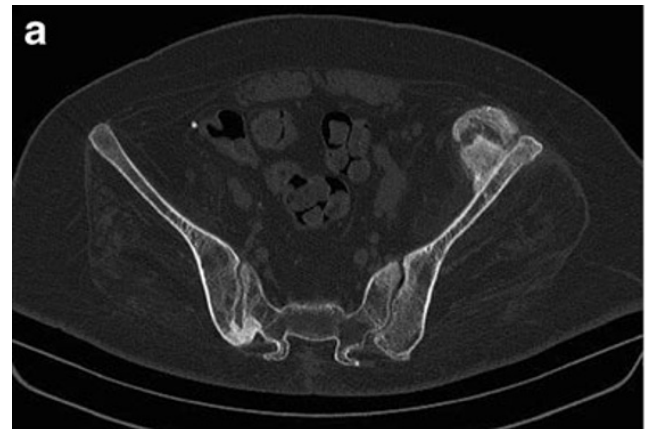

Fig. 12 A 30-year-old male patient affected by posterior element incomplete fusion (spina bifida occulta). a On first CT axial scan a bulky immature ossification is revealed adjacent to the left iliac wing. This HO was not comprised into the MR acquisition volume that was lower centred because of the clinical relevancy of a posterior

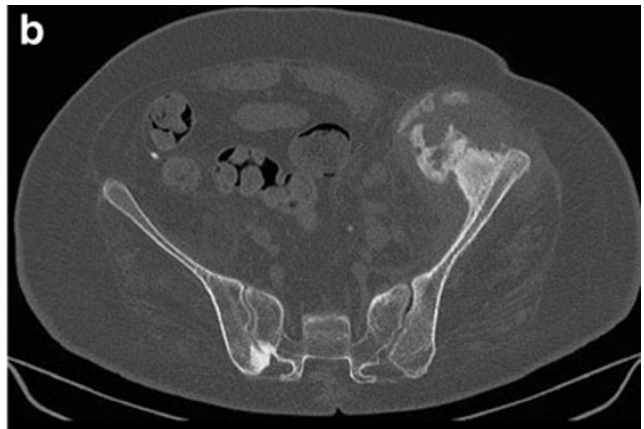

ischiofemural abscess. b A 10-month follow-up CT scan shows the lesion appears to be fragmented and partially resorbed. A marked soft tissue swelling is now visible caused by an underlying infectious process 
CT scan follow-up is advisable. In advanced ossification, imaging is key to correctly time surgical resection.

Open Access This article is distributed under the terms of the Creative Commons Attribution License which permits any use, distribution, and reproduction in any medium, provided the original author(s) and the source are credited.

\section{References}

1. Seipel R, Langner S, Platz T et al (2011) Neurogenic heterotopic ossification:epidemiology and morphology on conventional radiographs in an early neurological rehabilitation population. Skelet Radiol 28:151-160

2. Ledermann HP, Schweitzer ME, Morrison WB et al (2002) Pelvic heterotopic ossification: MR imaging characteristics. Radiology 222:189-195

3. Wick L, Berger M, Knecht $\mathrm{H}$ et al (2005) Magnetic resonance signal alterations in the acute onset of heterotopic ossification in patients with spinal cord injury. Eur Radiol 15:1867-1875

4. Mc Carthy EF, Sundaram M (2005) Heterotopic ossification: a review. Skelet Radiol 34:609-619

5. Wharton GW, Morgan TH (1975) Heteropic ossification. J Bone Joint Surg Am 52:105-112
6. Freed JH, Hahn H, Menter R et al (1982) The use of the three-phase bone scan in the early diagnosis of heterotopic ossification (HO) and in the evaluation of Didronel therapy. Paraplegia 20:208-216

7. Bressler EL, Marn CS, Gore RM et al (1987) Evaluation of ectopic bone by CT. Am J Roentgenol 148:931-935

8. Kransdorf MJ, Meis JM, Jeilinek JS (1991) Myositis ossificans:MR appearance with radiologic-pathologic correlation. AJR Am J Roentgenol 157:1243-1248

9. De Smet AA, Norris MA, Fisher DR et al (1992) Magnetic resonance imaging of myositis ossificans: analysis of seven cases. Skelet Radiol 21:503-507

10. Shirkhoda A, Armin AR, Bis KG et al (1995) MR imaging of myositis ossificans: variable patterns at different stages. J Magn Reson Imaging 5:287-292

11. Rossier AB, Bussat P, Infante F et al (1973) Current fact of paraosteo-arthropathy POA. Paraplegia 11:38-78

12. Huang AB, Schweitzer ME, Hume E et al (1998) Osteomyelitis of the pelvis/hips in paralyzed patients: accuracy and clinical utility of MRI. J Comput Assist Tomogr 22:437-443

13. Subhawong TK, Fishman E, Swart J et al (2010) Soft-tissue masses and masslike conditions: what does CT add to diagnosis and management? AJR Am J Roentgenol 194:1559-1567

14. Turecki MB, Taljanovic MS, Stubbs AY et al (2010) Imaging of musculoskeletal soft tissue infections. Skelet Radiol 39:957-971

15. Thomas BJ, Amstutz HC (1987) Prevention of heterotopic bone formation: clinical experience with diphosphonates. Hip 1987:59-69

16. Wharton GW, Morgan TH (1970) Ankylosis in the paralyzed patient. J Bone Joint Surg Am 52:105-112 